\title{
Improved preparation and identification of aristolochic acid-DNA adducts by solid-phase extraction with liquid chromatography-tandem mass spectrometry
}

\author{
GAO Haiyan ${ }^{1,2}$, GUO Fangqiu ${ }^{2}$, FENG Feng ${ }^{1}$, YIN Junfa ${ }^{1}$, SONG Maoyong ${ }^{1}$, WANG Hailin ${ }^{1, *}$ \\ 1. State Key Laboratory of Environmental Chemistry and Ecotoxicology, Research Center for Eco-Environmental Sciences, Chinese Academy of \\ Sciences, Beijing 100085, China. E-mail: hlwang@rcees.ac.cn \\ 2. Research Center of Modernization of Chinese Medicines, Central South University, Changsha 410083, China
}

Received 13 January 2009; revised 10 April 2009; accepted 14 April 2009

\begin{abstract}
Aristolochic acid (AA) is a known nephrotoxin and potential carcinogen, which can form covalent DNA adducts after metabolic activation in vivo and in vitro. A simple method for preparation and characterization of aristolochic acid-DNA adducts was developed. Four AA-adducts were synthesized by a direct reaction of AAI/AAII with 2'-deoxynucleosides. The reaction mixture was first cleaned-up and pre-concentrated using solid phase extraction (SPE), and further purified by a reversed-phase high performance liquid chromatography (HPLC). By the application of developed SPE procedure, matrices and byproducts in reaction mixture could be greatly reduced and adducts of high purity (more than $94 \%$ as indicated by HPLC) were obtained. The purified AA-DNA adducts were identified and characterized with liquid-electrospray ionization-quadrupole-time of flight-mass spectrometry (LC-ESI-Q-TOF-MS/MS) and LC-Diode array detector-fluorescence (LC-DAD-FL) analysis. This work provides a robust tool for possible large-scale preparation of AA-DNA adduct standards, which can promote the further studies on carcinogenic and mutagenic mechanism of aristolochic acids.
\end{abstract}

Key words: aristolochic acid; DNA adducts; solid phase extraction; quadrupole; time-of-flight tandem mass spectrometry

DOI: $10.1016 / \mathrm{S} 1001-0742(08) 62486-9$

\section{Introduction}

Aristolochic acid (AA) is one of structurally related nitrophenanthrene carboxylic acid derivatives. It is mainly produced in Aristolochia and Asarum plants (Mix et al., 1982; Bieler et al., 1997). Among AA analogues, AAI and AAII are the two major components (Schmeiser et al., 1996). AA-containing herbs had long been used to treat tumors, snake bites, obstetrics, rheumatism, small pox, and pneumonia (Kupchan and Doskotch, 1962; Rucker and Chung, 1975) until AAI and AAII were identified as potential carcinogens in rats (Schmeiser et al., 1986; Kohara et al., 2002; Chan and Cai, 2008; Mei et al., 2006) and nephrotoxic to rodents (Mengs, 1987, 1988; Mengs and Stotzem, 1993). Recently, AA has proven to be a risk factor of both aristolochic acid nephropathy (AAN) and Balkan endemic nephropathy (BEN) (Vanherweghem et al., 1993; Debelle et al., 2008; Grollman et al., 2007; Domijan and Sarić, 2008; Arlt et al., 2002; Stefanovic et al., 2006; Stiborova et al., 2008). It has been confirmed that when aristolochic acid is uptaken in human body, it can react with DNA to form covalent dA-AAI, dA-AAII, dGAAI and dG-AAII adducts, and etc. (Scheme 1) (Chan et al., 2007; Pfau et al., 1990). Therefore, AA-DNA adducts

\footnotetext{
* Corresponding author. E-mail: hlwang@rcees.ac.cn
}

can be used as biomarkers of organism exposure to AA and for the study of the mutagenic and carcinogenic properties of AA.

Since the AA-DNA adducts standards are still not commercially available and required to be prepared in individual laboratories, comprehensive researches on mutagenesis and carcinogenesis of AA have been restricted. For this purpose, several in vitro synthesis methods have been developed, including enzymatic activation and chemical activation (Pfau et al., 1990; Dong et al., 2006; Ji et al., 2008). Schmeiser et al. (1997) showed that the chemical activation by a large excess of zinc-dust in gently acidic conditions was by far the most efficient system for AAs. Due to its relatively high yields and simplicity, the chemical activation has becoming a promising method in the synthesis of AA-DNA adducts.

Besides the improvement of the synthesis, the sample enrichment and clean-up is also important for the preparation of high-purity AA-DNA adducts. However, the sample pretreatment has not well optimized yet. Previously, single AA-adducted nucleosides were usually obtained by reaction of AA with 2'-deoxynucleosides followed by liquid-liquid extraction (LLE) (Chan et al., 2007; Schmeiser et al., 1997). In these procedures, high-purity of adducts can not be obtained and a large volume of organic solvent 

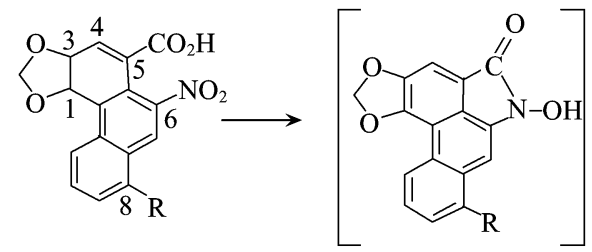

Aristolochic I (AAI) : $\mathrm{R}=\mathrm{OCH}_{3} \quad \mathrm{~N}$-Hydroxyaristolactam Aristolochic II (AAII) : $\mathrm{R}=\mathrm{H}$

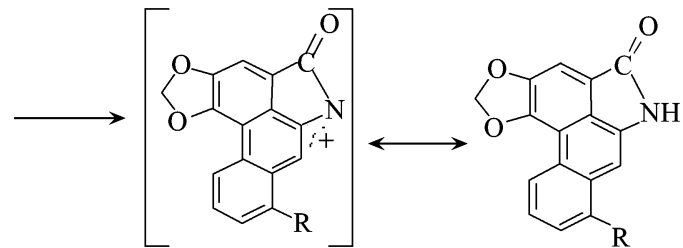

Aristolactam nitrenium ion

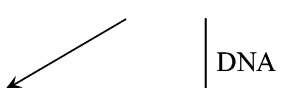
$\downarrow$ DNA
Aristolactam I (AAI) : $\mathrm{R}=\mathrm{OCH}_{3}$

Aristolactam II (AAII) : $\mathrm{R}=\mathrm{H}$<smiles>[R]c1cccc2c(O)c3c=2c2c4c(cc(c12)C(=O)N3)OCO4</smiles>

7-Hydroxyaristolactam
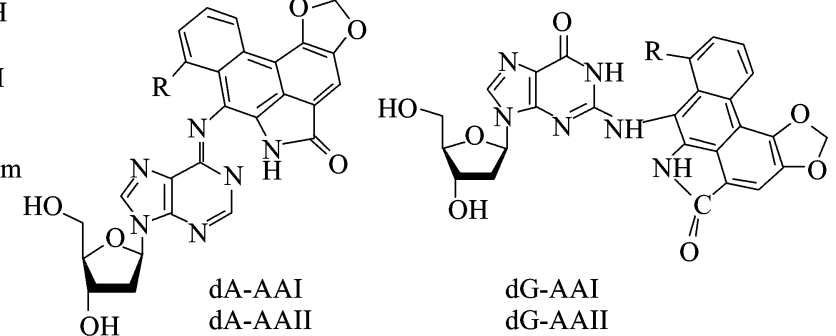

dG-AAI

dG-AAII

Scheme. 1 Proposed pathway for the aristolochic acid (AA)-derived DNA adducts formation. For aristolactam I (AAI) R represents as $\mathrm{OCH}_{3}$, and as $\mathrm{H}$ for aristolactam II (AAII).

is needed. This is somewhat tedious and time-consuming, making the sample pretreatment inefficient. Solid-phase extraction (SPE) protocol appears to be a good alternative to LLE. SPE uses disposable columns packed with small amounts of liquid chromatographic sorbent to clean-up reaction mixtures. Compared with LLE, by changing the elution conditions, ideal selectivity and high-purity of target analytes can be easily achieved by SPE. This greatly facilitates the isolation and purification of the individual DNA adduct (Feng et al., 2008; Gaskell et al., 2007).

Following the synthesis and preparation, the identification and characterization of the AA-DNA adducts are required. This can be achieved by liquid chromatography coupled with tandem mass spectrometry (LC-MS/MS) by taking advantages of its ability to provide structural information (Goldman et al., 2007; Singh and Farmer, 2006; Flarakos et al., 2005). Moreover, the LC-MS/MS method can be performed with high sensitivity and without potential health hazard, compared with the popular ${ }^{32} \mathrm{P}$ postlabeling for assaying AA-DNA adducts (Dong et al., 2006; Arlt et al., 2001; Shibutani et al., 2007; Chan et al., 2008).

In this study, we demonstrate a simple method for chemical synthesis, optimum preparation and characterization of AA-adducts. The chemically synthesized AA-nucleosides were cleaned up and isolated using SPE, and further purified by an optimized reversed-phase HPLC. The chemical identities of the AA-adducts were subtly distinguished by LC-diode array detector-fluorescence (LC-DAD-FL) and LC-electrospray-tandem mass spectrometry (LC-ESIMS/MS) detection.

\section{Materials and methods}

\subsection{Chemicals and reagents}

Aristolochic acid I (AAI) and deoxyguanosine (dG) were purchased from Acros (Morris Plains, NJ, USA).
Deoxyadenosine (dA) was obtained from Sigma (St. Louis, USA). Aristolochic acid II (AAII) was purchased from AppliChem (Darmstadt, Germany). Acetonitrile and methanol were of HPLC grade and purchased from Fisher Scientific (Pittsburgh, USA). Ultrapure water (18.2 M $\Omega$ ) was prepared by an ELGA ultra-pure water system (ELGA, High Wycombe, UK). Other chemicals are used of HPLC or analytical grade. It should be notice that AAI and AAII are both mutagen and carcinogen, and caution should be taken when handling these compounds.

\subsection{Apparatus}

A Hitachi L-2000 HPLC system consists of a L-2130 low-pressure gradient pump, a L-2200 autosampler, a L-2455 diode array detector (DAD) and a L-2485 fluorescence detector (FL) (Hitachi, Tokyo, Japan). An EZChrom Elite chromatography data system was used for the operation and data acquisition.

High performance liquid chromatography-electrospray ionization-quadrupole time-of-flight tandem mass spectrometry (HPLC-ESI-Q-TOF-MS/MS) system comprises an Alliance 2695 HPLC (Waters, UK) and a Waters Micromass Quattro Micro (Milford, USA) tandem quadrupole mass spectrometer, which is equipped with an electrospray ionization (ESI) source. Data acquisition was conducted by MassLynx 4.0 software (Waters, MA, USA).

\subsection{Synthesis of AA-DNA adducts}

DNA adducts including dA-AAI, dA-AAII, dG-AAI and dG-AAII were synthesized by incubating deoxynucleosides ( $\mathrm{dG}$ or $\mathrm{dA}$ ) with AA (AAI or AAII) according to the method described by Schmeiser et al. (1997). The dG/dA solution $(2 \mathrm{mg}$, dissolved in $1 \mathrm{~mL}$ of $50 \mathrm{mmol} / \mathrm{L}$ potassium phosphate buffer ( $\mathrm{pH} 5.8$ )), was mixed with AAI/AAII solution ( $0.8 \mathrm{mg}$ in $30 \mu \mathrm{L}$ of dimethylformamide), then 20 mg zinc powder was added. The reaction mixture was then incubated for $16 \mathrm{~h}$ at $37^{\circ} \mathrm{C}$. At last, the reaction mixture 
was then centrifuged at $12000 \times g$ for 10 min to remove the zinc residues.

\subsection{SPE enrichment and cleanup}

A SPE protocol was developed for the removal of complex matrix interferences in reaction mixtures. First, the supernatant was loaded onto a Clearnet $\mathrm{C} 18$ cartridge (100 mg, $1 \mathrm{~mL}$ ), which was preconditioned with $2 \mathrm{~mL}$ of methanol and $2 \mathrm{~mL}$ of deionized water in sequence. Then, the cartridge was rinsed with $1 \mathrm{~mL}$ of deionized water twice, and sequentially washed with $1 \mathrm{~mL}$ of $20 \%, 30 \%$, $40 \%$ and $50 \%$ of methanol solution. Finally, the cartridge was eluted by $1 \mathrm{~mL}$ of methanol twice. The collected SPE fractions were subjected to HPLC-DAD-FL detection to monitor target products and to evaluate cleanup efficacy.

The SPE procedure was compared with LLE to obtain an efficient pretreatment. Briefly, the reaction mixture was split into two equal portions. One portion was pretreated by LLE and the other by SPE. The LLE procedure was performed by the method described by Chan et al. (2007) and the SPE was carried out as described above.

\subsection{HPLC preparation of four AA-DNA adducts}

The collected SPE fractions containing AA-DNA adducts were subjected to further purification to prepare AA-DNA adducts standards with appropriate purity. The samples were separated using the Hitachi L-2000 series HPLC instrument. The Elite Hypersil BDS C18 column (4.6 mm i.d. $\times 250 \mathrm{~mm}$ length, $5 \mu \mathrm{m}$ thickness) was used to analyze and purify each AA-DNA adducts. The mobile phase consists of acetonitrile-water $(0.2 \%$ acetic acid) $(30: 70, V / V)$. The flow rate was $1.2 \mathrm{~mL} / \mathrm{min}$ and column temperature was kept at $25^{\circ} \mathrm{C}$. The injection volume was $10 \mu \mathrm{L}$ for analytical separation and $99 \mu \mathrm{L}$ for LC preparation. The wavelength of the DAD detector was set at $260 \mathrm{~nm}$. The excitation and emission wavelengths of fluorescence were 304 and $480 \mathrm{~nm}$, respectively. Collected fractions were dried using lyophilization and stored at $-20^{\circ} \mathrm{C}$.

\subsection{HPLC-ESI-Q-TOF-MS/MS analysis}

The HPLC-MS/MS analysis of the four AA-DNA adducts was conducted under the selected chromatographic conditions as described in Section 1.5. The mass spectrometer was operated in positive ionization mode. The effluent from the HPLC column was split into a stainless capillary at $0.3 \mathrm{~mL} / \mathrm{min}$ for Q-TOF-MS/MS analysis. The mass range was from $\mathrm{m} / \mathrm{z} 120$ to 800 . The cone voltage was $30.0 \mathrm{~V}$, the collision energy was 5.0 $\mathrm{V}$ for LC-MS analysis and the capillary voltage was maintained at $3.5 \mathrm{kV}$. Nitrogen was used as a nebulizer gas. For collision-activated dissociation (CAD) MS/MS fragmentation analysis, argon was used as the collision gas. The collision energy was $25 \mathrm{~V}$, and the gas pressure was kept at $0.3 \mathrm{MPa}$.

\section{Results and discussion}

\subsection{Synthesis and preconcentration of AA-DNA adducts}

Reduction of AAI by zinc powder in the present of $2^{\prime}$-deoxyguanosine leads to the formation of dG-AAI adduct. The overall yield of dG-AAI adduct is up to $2.0 \%$. Compared with that xanthine oxidase-activated in vitro reaction of AA with calf thymus DNA $(0.1 \%-0.4 \%)$ (Pfau et al., 1990), the yield was greatly increased by about 10 fold. The used method exhibits several advantages, such as high yield, gentle reaction conditions, no need for enzyme, low cost, and so on. Therefore, it is possible to scale up the synthesis of AA-adducts by using the chemical reduction.

Sample cleanup procedure plays an important role in the elimination of matrix effects and improvement of determination sensitivity. Solid phase extraction is a powerful sample preparation technique currently available for rapid clean-up and enrichment of sample analytes preceding chromatographic analysis. In order to eliminate the matrices efficiently, SPE cleanup was conducted on a Cleanert C18 cartridge. Figure 1 illustrates SPE removal of matrices and extraction of dG-AAI adducts. It is evident that unreacted $\mathrm{dG}$ and byproducts can be removed after
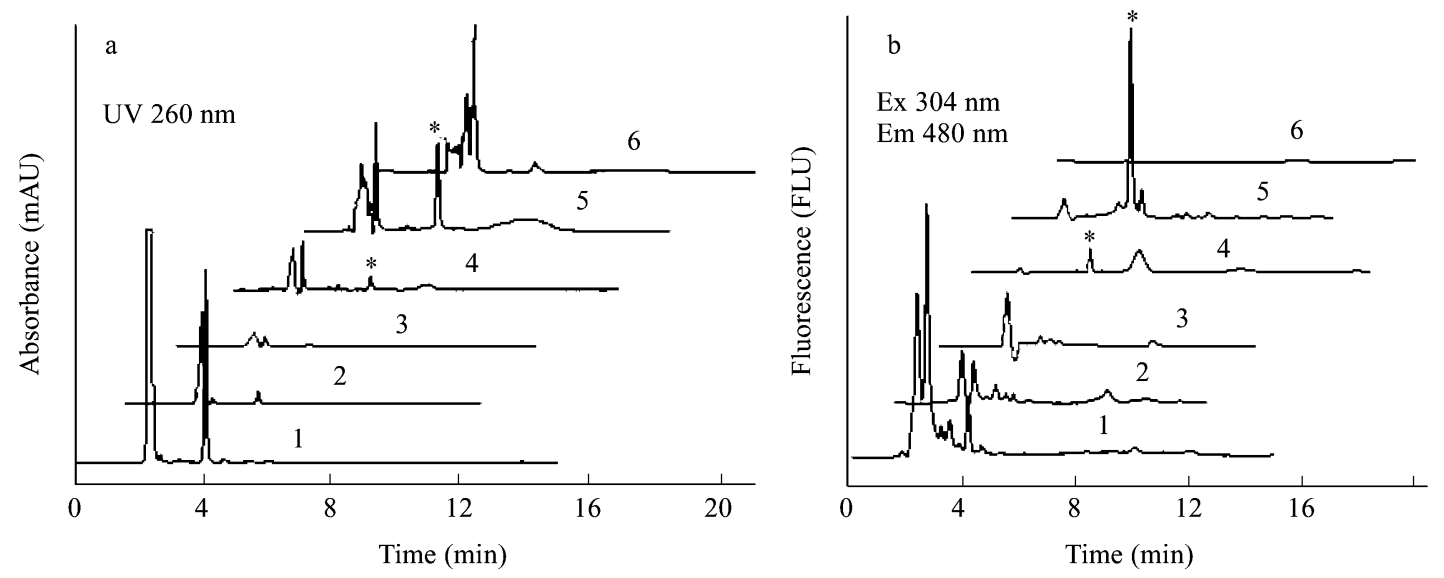

Fig. 1 HPLC-DAD-FL analysis of each solid-phase extraction (SPE) fraction obtained from the reaction of deoxyguanosine (dG) with AAI. UV absorbance (a) was monitored at $260 \mathrm{~nm}$ and fluorescence response (b) was monitored at Ex $304 \mathrm{~nm}$, Em $480 \mathrm{~nm}$. Methanol solution (1) 20\%, (2) 30\%, (3) $40 \%$, (4) $50 \%$; (5) methanol elution; (6) second methanol elution. 
washing with $2 \mathrm{~mL}$ deionized water, and $1 \mathrm{~mL}$ of $20 \%$, $30 \%$ or $40 \%$ of methanol solution. During these washing steps, no significant loss of dG-AAI adducts was observed. It also can be seen from Fig. 1 that the AA-adducts can be partially eluted out by $50 \%$ of methanol solution, indicating that $40 \%$ of methanol solution is good for cleaning up the matrices and retaining the target products.

The retained adducts were eluted from the cartridge by $1 \mathrm{~mL}$ of pure methanol. No adducts was detected in the eluate by using additional $1 \mathrm{~mL}$ of methanol. The result indicates that $\mathrm{dG}-\mathrm{AAI}$ adducts can be eluted completely by $1 \mathrm{~mL}$ of methanol. According to the above observations, the optimized SPE protocol was developed as follows: the cartridge loaded by reaction mixture $(1 \mathrm{~mL})$ was washed with $1 \mathrm{~mL}$ deionized water twice, followed with $1 \mathrm{~mL}$ of $40 \%$ methanol solution 3 times, and then eluted with $1 \mathrm{~mL}$ methanol. The collected fractions were evaporated under a gentle stream of nitrogen and residues were redissolved in $100 \mu \mathrm{L}$ methanol before LC-DAD-FL analysis.

Figure 2 shows the chromatograms obtained at 260 $\mathrm{nm}$ from the reaction mixtures before and after clean-up. Before SPE procedure, there were a plenty of matrices and byproducts. Whereas it is apparent that most interference peaks can be removed by SPE clean-up.

The optimized SPE protocol was also applicable for dG-AAII, dA-AAI and dA-AAI adducts (data not shown). Since most matrices and byproducts were eliminated, the SPE protocol greatly facilitated the further purification of each AA-adduct by HPLC.

To meet the objectives for complete clean-up of sample matrices, the performance of SPE and LLE were compared. Despite of using triplicate organic solvent extraction, the recovery of LLE is poor $(9.40 \%)$. In contrast, the recovery of SPE is $88.9 \%$. Moreover, the consumption of organic solvent by LLE was one time larger than that by SPE. It is evident that the developed SPE protocol not only exhibits a higher enrichment efficiency and less consumed volume of organic solvents, but also displays higher separation selectivity. The SPE procedure applied here allows the removal of the byproducts and unreacted deoxyguanosine with which may interfere the preparation of AA-adducts and subsequent LC-MS/MS analysis.

\subsection{Purification of AA-DNA adducts}

The AA-DNA adducts extracted from the reaction mixtures by SPE procedure were further purified using HPLC. Except for the injection volume increased from $10 \mu \mathrm{L}$ for analysis to $99 \mu \mathrm{L}$ for preparation, the mobile phase also varied with different AA-DNA adducts. The concentrations of acetonitrile were $40 \%$ for dA-AAI, $30 \%$ for dG-AAI as well as dA-AAII, and $25 \%$ for dG-AAII. Under such conditions, the four AA-DNA adducts were well separated and then were collected. As shown in Fig. 3, after SPE procedure the matrices and byproducts in the reaction mixtures of dA-AAI, dG-AAI and dA-AAII were greatly removed, however, for dG-AAII, there were still tiny interferences after SPE.

The purity of each product was further determined by HPLC-DAD-FL. It can be seen from Fig. 4 that all of the four purified AA-DNA adducts are of high purity $(>94.4 \%)$.

\subsection{HPLC-DAD-FL analysis of AA-DNA adducts}

As a class of structurally related phenanthrene derivatives, all AA-DNA adducts have suitable UV absorbance which enables them to be determined by UV absorbance. Moreover, being rigid polycyclic aromatics, aristolactams (ALs) have a strong fluorescence, and thus the AA-DNA adducts could also be detected by fluorescence detection. Therefore, the hyphenation of DAD and FL should be a useful detection tool for the simultaneous analysis of the AA-DNA adducts. The four adducts all have UV $260 \mathrm{~nm}$ absorbance and three of them have strong fluorescence response except dG-AAII. The $\mathrm{dG}$ and $\mathrm{dA}$ adducts of AAI have longer retention times than that of their corresponding AAII adducts, which is probably caused by the methoxy group binding to $\mathrm{C} 8$. Moreover, the $\mathrm{dG}$ adducts of AAI and AAII are more polar and thus have shorter retention times than their corresponding $\mathrm{dA}$ adducts. Therefore, it is reasonable that under the conditions described above the four AA-DNA adducts are eluted in the order of dG-AAII, dG-AAI, dA-AAII and dA-AAI (Fig. 4).

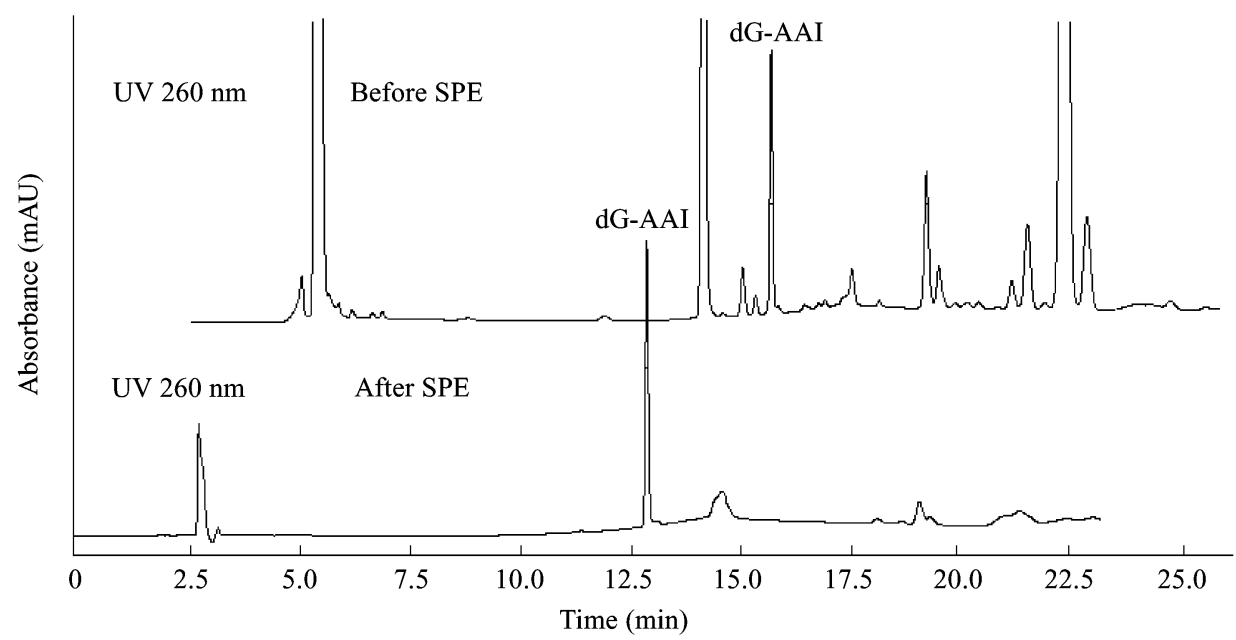

Fig. 2 Chromatograms of AAI-dG reaction mixtures before and after SPE procedure. 

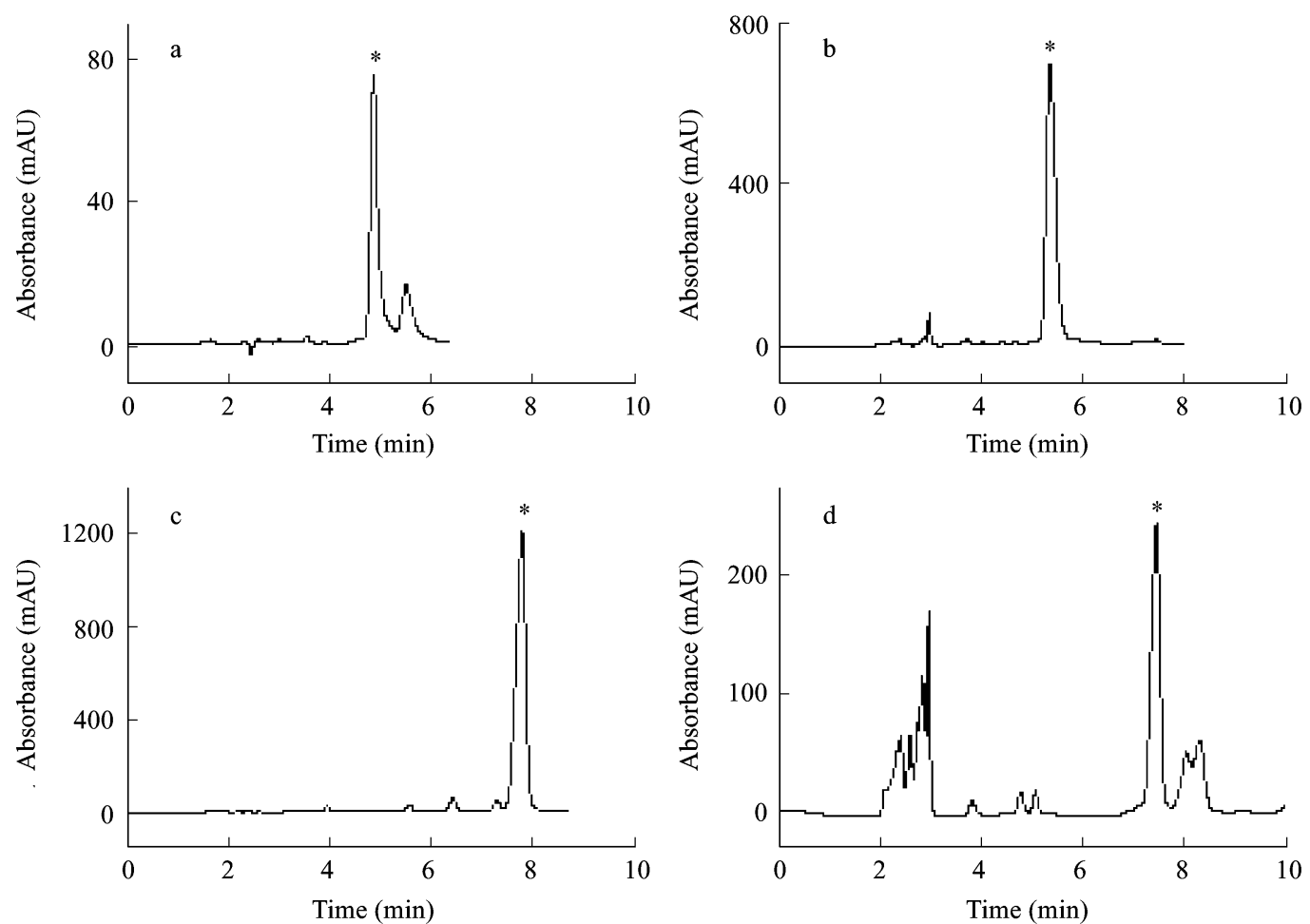

Fig. 3 HPLC preparation of AA-DNA adducts including dA-AAI (a), dG-AAI (b), dA-AAII (c) and dG-AAII (d). Mobile phase for dA-AAI was $40 \%$ acetonitrile-water $(0.2 \%$ acetic acid) $(V / V)$, for dG-AAI, dA-AAII was $30 \%$ acetonitrile-water $(0.2 \%$ acetic acid) $(V / V)$, and for dG-AAII was $25 \%$ acetonitrile-water $(0.2 \%$ acetic acid) $(V / V)$.
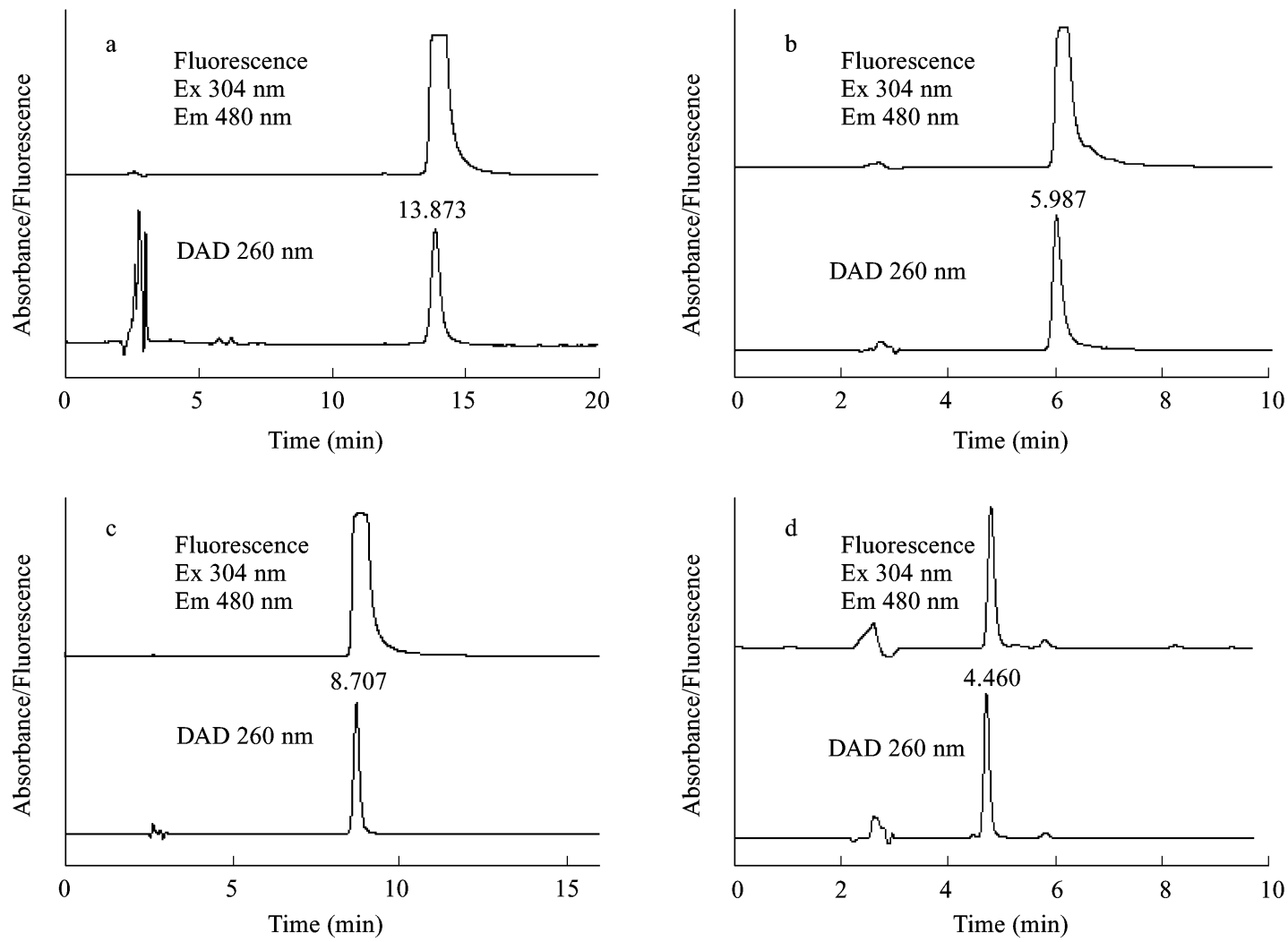

Fig. 4 HPLC analysis single AA-DNA adducts including dA-AAI (a), dG-AAI (b), dA-AAII (c) and dG-AAII (d) purified by the Hypersil C18 column. $\mathrm{UV}$ absorbance was monitored at $260 \mathrm{~nm}$ and fluorescence response (b) was monitored at Ex $304 \mathrm{~nm}$, Em $480 \mathrm{~nm}$. 


\subsection{Characterization and identification of AA-DNA} adducts

The AA-DNA adducts were characterized by a combination of UV spectroscopy and HPLC-MS/MS. The on-line UV spectra were recorded in the range of 200$500 \mathrm{~nm}$. The absorbance spectra are shown in Fig. 5 and the characteristic absorbance wavelength for each adduct is listed in Table 1. Interestingly, two types of absorbance spectra were observed. The UV absorbance spectrum of dA-AAI and dG-AAI are similar to the spectra of aristolactam I (LI) while the UV absorbance spectra of dA-AAII and dG-AAII are similar to the spectra of

Table 1 Characteristic of UV absorbencies of the four AA-DNA adducts

\begin{tabular}{ll}
\hline AA-DNA adduct & UV (nm) \\
\hline dA-AAI & $246,304,411$ \\
dG-AAI & $247,258,299,410$ \\
dA-AAII & $226,265,291,393$ \\
dG-AAII & $233,264,291,393$ \\
\hline
\end{tabular}

aristolactam II (LII), which suggests that the aristolochic acid moiety in the adducts contains the lactam structure. The differences may be mainly attributed to the methoxy groups. The UV absorbance spectrum of the adducts of AAI showed maxima at 246-247, 299-304 and 410-411 $\mathrm{nm}$ while the adducts of AAII exhibited the characteristic absorbance at 264-265, 291, and $393 \mathrm{~nm}$.

The AA-DNA adducts were then subjected to LC-ESIQ-TOF-MS analysis. The MS conditions were performed in the positive ion mode, and acetic acid was added to mobile phase to facilitate ionization. The abundance of protonated molecular ion peak, $[\mathrm{M}+\mathrm{H}]^{+}$peaks of adducts dG-AAI and dG-AAII at $m / z 559$ and 529 were very low, whereas the major fragment ion peaks at $m / z 443$ and 413 were relatively high, which resulted from the neutral loss of deoxyribose moiety. However, for dA-AAI and dAAAII, the abundance of protonated molecular ion peak at $m / z 543$ and 513 were much higher than the fragment ion peak at $m / z, 427$ and 397. This indicated that dG-AA adducts had weak stability when structurally compared with dA-AA adducts.

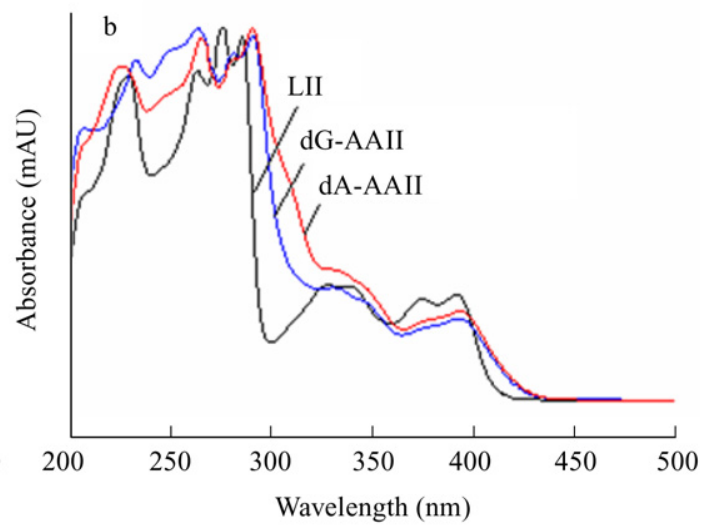

Fig. 5 UV absorbance spectra of LI, LII and AA-DNA adducts (dA-AAI, dG-AAI, dA-AAII, and dG-AAII). The spectra were extracted from HPLCDAD-FL analysis of purification of adducts.
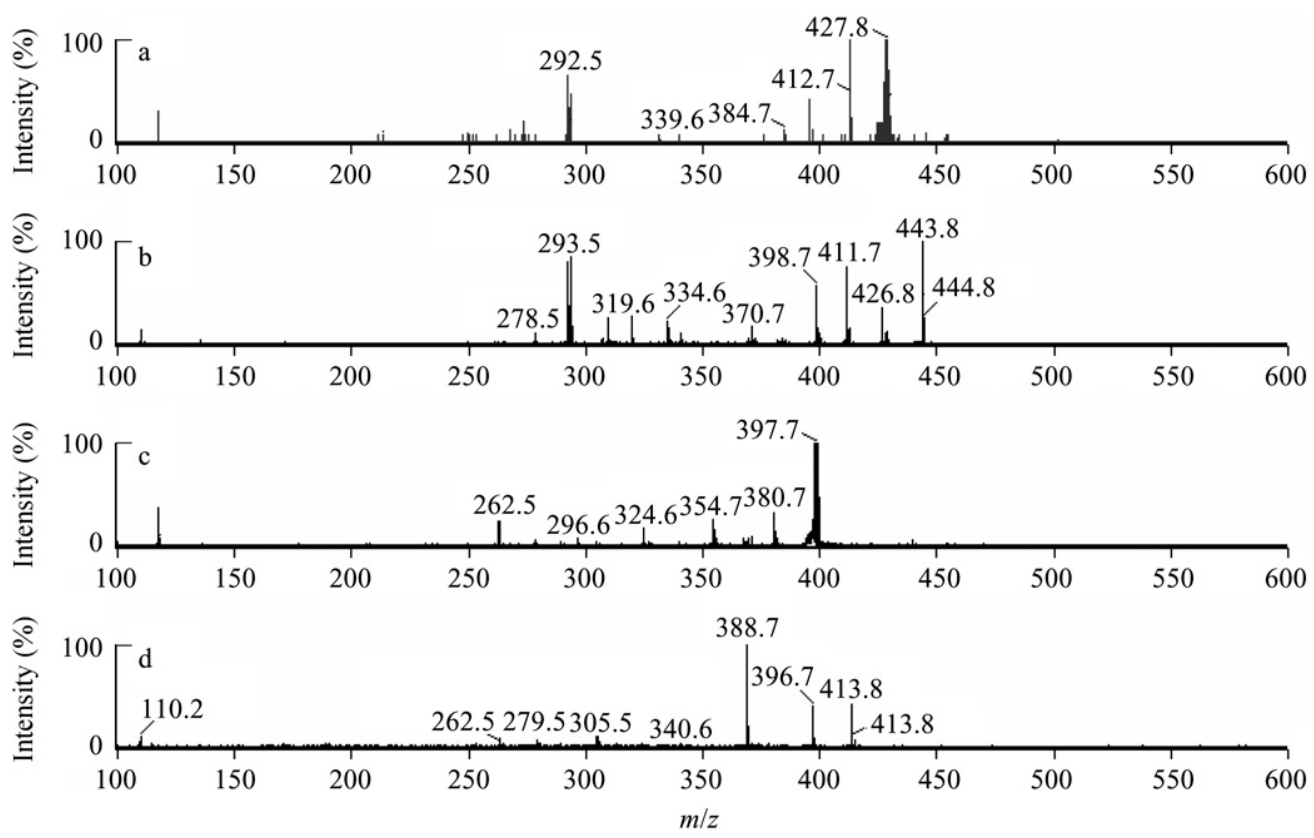

Fig. 6 MS-MS spectra of the $[\mathrm{M}+\mathrm{H}]^{+}$adduct ions of dA-AAI (a), dG-AAI (b), dA-AAII (c), and dG-AAII (d). 
Further structural information of AA-DNA adducts was obtained by LC-MS/MS analysis. The product ion spectrum of the AA-DNA adducts exhibited characteristic fragmentation pattern with the loss of $116 \mathrm{Da}$, which resulted from internal hydrogen transfer and fragmentation loss of a deoxyribose moiety (Fig. 6). Aristolactam moieties are corresponding to $\mathrm{m} / \mathrm{z} 262$ and 292 for aristolactam II and aristolactam I, respectively.

\section{Conclusions}

In this work, four AA-DNA adducts were prepared by an optimized SPE procedure coupled with HPLCDAD purification method. An identification and analysis method for these adducts by LC-DAD-FL and LC-QTOF-MS/MS was also presented. By means of SPE, the AA-DNA adducts were purified preliminarily, which facilitated further the preparation of AA-adducts by HPLC. High-purity of adducts were obtained after the further HPLC purification. The described SPE procedure may be promising for a general clean-up in the analysis of AADNA adducts.

\section{Acknowledgments}

This work was financially supported by the National Basic Research Program (973) of China (No. 2007CB407305, 2008CB417201), the National High Technology Research and Development Program (863) of China (No. 2007AA06A407) and the National Natural Science Foundation of China (No. 20737003, 20621703, 20805057).

\section{References}

Arlt V M, Ferluga D, Stiborova M, Pfohl-Leszkowicz A, Vukelic $\mathrm{M}, \mathrm{Ceovic} \mathrm{S}$ et al., 2002. Is aristolochic acid a risk factor for Balkan endemic nephropathy-associated urothelial cancer? International Journal of Cancer, 101(5): 500-502.

Arlt V M, Pfohl-Leszkowicz A, Cosyns J P, Schmeiser H H, 2001. Analyses of DNA adducts formed by ochratoxin A and aristolochic acid in patients with Chinese herbs nephropathy. Mutation Research, 494(1-2): 143-150.

Bieler C A, Stiborova M, Wiessler M, Cosyns J P, Strihou C Y, Schmeiser H H, 1997. ${ }^{32} \mathrm{P}$-postlabelling analysis of DNA adducts formed by aristolochic acid in tissues from patients with Chinese herbs nephropathy. Carcinogenesis, 18(5): 1063-1067.

Chan W, Zheng Y, Cai Z W, 2007. Liquid chromatographytandem mass spectrometry analysis of the DNA adducts of aristolochic acids. Journal of the American Society for Mass Spectrometry, 18(4): 642-650.

Chan W, Lee K C, Liu N, Cai Z W, 2007. A sensitivity enhanced high-performance liquid chromatography fluorescence method for the detection of nephrotoxic and carcinogenic aristolochic acid in herbal medicines. Journal of Chromatography A, 1164(1-2): 113-119.

Chan W, Cai Z W, 2008. Aristolochic acid induced changes in the metabolic profile of rat urine. Journal of Pharmaceutical and Biomedical Analysis, 46(4): 757-762.

Chan W, Yue H, Poon W T, Chan Y W, Schmitz O J, Kwong D W et al., 2008. Quantification of aristolochic acidderived DNA adducts in rat kidney and liver by using liquid chromatography-electrospray ionization mass spectrometry. Mutation Research, 646(1-2): 17-24.

Debelle F D, Vanherweghem J L, Nortier J L, 2008. Aristolochic acid nephropathy: A worldwide problem. Kidney International, 74(2): 158-169.

Domijan A M, Sarić M, 2008. Mycotoxic and aristolochic acid theories of the development of endemic nephropathy. Arh Hig Rada Toksikol, 59(1): 59-65.

Dong H, Suzuki N, Torres M C, Bonala R R, Johnson F, Grollman A P, Shibutani S, 2006. Quantitative determination of aristolochic acid-derived DNA adducts in rats using ${ }^{32} \mathrm{P}$ postlabelling/polyacrylamide gel electrophoresis analysis. Drug Metabolism and Disposition, 34(7): 1122-1127.

Feng F, Yin J F, Song M Y, Wang H L, 2008. Preparation, identification and analysis of stereoisomeric anti-benzo[a]pyrene diol epoxide-deoxyguanosine adducts using phenyl liquid chromatography with diode array, fluorescence and tandem mass spectrometry detection. Journal of Chromatography A, 1183(1-2): 119-128.

Flarakos J, Xiong W N, Glick J, Vouros P, 2005. A deoxynucleotide derivatization methodology for improving LC-ESI-MS detection. Analytical Chemistry, 77: 23732380.

Gaskell M, Kaur B, Farmer P B, Singh R, 2007. Detection of phosphodiester adducts formed by the reaction of benzo[a]pyrene diol epoxide with 2'-deoxynucleotides using collision-induced dissociation electrospray ionization tandem mass spectrometry. Nucleic Acids Research, 35(15): 5014-5027.

Grollman A P, Shibutani S, Moriya M, Miller F, Wu L, Moll U et al., 2007. Aristolochic acid and the etiology of endemic (Balkan) nephropathy. Proceedings of the National Academy of Sciences of the United States of American, 104(29): 12129-12134.

Ji W X, Liu M X, Yang C D, Chen Y P, 2008. Synthesis and mass spectrometric analysis of aristolochic acid-deoxyguanosine adducts. Acta Pharmaceutica Sinica, 43(3): 295-298.

Kohara A, Suzuki T, Honma M, Ohwada T, Hayashi M, 2002. Mutagenicity of aristolochic acid in the lambda/lacZ transgenic mouse (MutaMouse). Mutation Research, 515(1-2): 63-72.

Kupchan M S, Doskotch R W, 1962. Tumor inhibitors I, aristolochic acid, the active principle of Aristolochia indica. Journal of Medicinal and Pharmaceutical Chemistry, 5(3): 657-659.

Mei N, Arlt V M, Phillips D H, Heflich R H, Chen T, 2006. DNA adduct formation and mutation induction by aristolochic acid in rat kidney and liver. Mutation Research, 602(1-2): 83-91.

Mengs U, 1987. Acute toxicity of aristolochic acid in rodents. Archives of Toxicology, 59(5): 328-331.

Mengs U, 1988. Tumor induction in mice following exposure to aristolochic acid. Archives of Toxicology, 61(6): 504-505.

Mengs U, Stotzem C D, 1993. Renal toxicity of aristolochic acid in rats as an example of nephrotoxicity testing in routine toxicology. Archives of Toxicology, 67(5): 307-311.

Mix D B, Guinaudeau H, Sharmma M, 1982. The aristolochic acids and aristolactams. Journal of Natural Products, 45(6): 657-666.

Pfau W, Schmeiser H H, Wiessler M, 1990. Aristolochic acid binds covalently to exocyclic amino group of purine nucleotides in DNA. Carcinogenesis, 11(2): 313-319.

Rucker V G, Chung B S, 1975. Aristolochic acids from Aristolochia manshuriensis (author's transl). Planta Medica, 
27(1): 68-71.

Schmeiser H H, Bieter C A, Wiessler M, Strihou C Y, Cosyns J P, 1996. Detection of DNA adducts formed by aristolochic acid in renal tissue from patients with Chinese herbs nephropathy. Cancer Research, 56(9): 2025-2028.

Schmeiser H H, Pool B L, Wiessler M, 1986. Identification and mutagenicity of metabolites of aristolochic acid formed by rat liver. Carcinogenesis, 7(1): 59-63.

Schmeiser H H, Frei E, Wiessler M, Stiborova M, 1997. Comparison of DNA adduct formation by aristolochic acids in various in vitro activation systems by ${ }^{32} \mathrm{P}$-postlabelling: evidence for reductive activation by peroxidases. Carcinogenesis, 18(5): 1055-1062.

Shibutani S, Dong H, Suzuki N, Ueda S, Miller F, Grollman A P, 2007. Selective toxicity of aristolochic acids I and II. Drug Metabolism and Disposition, 35(7): 1217-1222.
Singh R, Farmer P B, 2006. Liquid chromatography-electrospray ionization-mass spectrometry: the future of DNA adduct detection. Carcinogenesis, 27(2): 178-196.

Stefanovic V, Toncheva D, Atanasova S, Polenakovic M, 2006. Etiology of Balkan endemic nephropathy and associated urothelial cancer. American Journal of Nephrology, 26(1): $1-11$.

Stiborova M, Frei E, Arlt V M, Schmeiser H H, 2008. Metabolic activation of carcinogenic aristolochic acid, a risk factor for Balkan endemic nephropathy. Mutation Research, 658(1-2): 55-67.

Vanherweghem J L, Depierreux M, Tielemans C, Abramowicz D, Dratwa M, Jadoul M et al., 1993. Rapidly progressive interstitial renal fibrosis in young women: association with slimming regimen including Chinese herbs. The Lancet, 341(8842): 387-391. 